\title{
ON THE EMBEDDING OF COMPLEMENTS OF SOME HYPERBOLIC PLANES II
}

\author{
PINAR ANAPA, İBRAHIM GÜNALTILI AND SÜKRÜ OLGUN
}

\begin{abstract}
In this paper, we studied that a linear space, which is the complement of linear space whose points are not on a pentagon, hexagon or a heptagon in a projective subplane of order $m$, is embeddable in an unique way in a projective plane of order $n$. In addition, we showed that this linear space is the complement of certain regular hyperbolic plane in the sense of Graves [5] with respect to a finite projective plane..
\end{abstract}

\section{INTRODUCTION}

The complementation problem with respect to a projective plane is the following : Remove a certain configuration of points and lines from the plane, determine the parameters of the resulting space. The problem of embedding the " complements " of various configuration in the projective planes has been studied by various authors ( $[1],[2],[3],[4],[5], \ldots$ ). In 1970, Dickey solved the problem for the case where the configuration removed was a unital [5]. In 1987, L. M. Batten characterized linear spaces which are the complements of affine or projective subplanes of finite projective planes and showed that these spaces can be embeddable in an unique way in a projective plane of order $n$ [1]. A generalized of Batten's Theorem [1] was given by Günaltılı and Olgun [7]. In [8], Günaltılı, Anapa and Olgun showed that a linear space, which is the complement of a linear space having points are not on a trilateral or a quadrilateral in a projective subplane of order $m$, is embeddable in an unique way in a projective plane of order $n$. In addition, it was determined that this linear space is the complement of certain regular hyperbolic plane in the sense of Graves [6] with respect to a finite projective plane.

In this study, we showed that a linear space, which is the complement of a linear space whose points are not on a pentagon, hexagon or a heptagon in a projective subplane of order $m$, is embeddable in a unique way in a projective plane of order $n$. In addition, we determined that this linear space is the complement of certain

Received by the editors April 09, 2007; Rev: Feb. 13, 2008 Accepted: March 20, 2008.

2000 Mathematics Subject Classification. Primary51E20,.; Secondary 51A45.

Key words and phrases. Linear space, projective plane, hyperbolic plane. 
regular hyperbolic plane in the sense of Graves [6] with respect to a finite projective plane.

Now, we give some definitions required.

Definition 1.1: A non-degenerate finite linear space is a pair $\mathcal{S}=(\mathcal{P}, \mathcal{L})$, where $\mathcal{P}$ is a finite set of points and $\mathcal{L}$ is a family of proper subsets of $\mathcal{P}$, which are called lines, such that: any two points are on unique line, each line has at least two points and there are at least two lines.

If $\mathcal{S}=(\mathcal{P}, \mathcal{L})$ is a finite linear space, that is, $|\mathcal{P}|<\infty$. The number of lines passing through a point $P$ is called the degree of $P$ and denoted by $b(P)$. The number of points on a line $l$ is called the degree of $l$ and denoted by $v(l) . v$ and $b$ denote the total number of points and lines of $\mathcal{S}$, respectively. The number $v, b, b(P)$ and $v(l)$ are called the parameters of $\mathcal{S}$.

The parameters of finite linear space $k_{m}, k_{M}, r_{m}, r_{M}$ are defined as stated below.

$k_{m}=\min \{v(l): l \in \mathcal{L}\}$

$k_{M}=\max \{v(l): l \in \mathcal{L}\}$

$r_{m}=\min \{b(P): P \in \mathcal{P}\}$

$r_{M}=\max \{b(P): P \in \mathcal{P}\}$

The term $i$-point or $i$-line may also used to refer respectively to a point or a line of degree $i$. Moreover; $b_{k}$ denotes the total number of $k$-lines, $v_{k}$ denotes the total number of $k$-points and $b_{k}(P)$ denotes the total number of $k$-lines passing through a point $P$.

The integer $n$ defined by $n+1=\max b(P): P \in \mathcal{P}\}$ is the " order " of the linear space.

Definition 1.2 : A finite $(m+1)$-regular hyperbolic plane $(\mathcal{P}, \mathcal{L})$, in the sense of Graves, is a non-trivial $(m+1)$-regular linear space such that :

H1 There are four points, no three of which are collinear

H2 If $P$ is a point not on a line $l$, then there exist at least two lines, not meeting $l$ and through $P$.

H3 If a subset $\mathcal{P}^{\prime}$ of the points of $\mathcal{P}$ contains three non-collinear points and contains all points on the lines through pairs of distinct points of $\mathcal{P}^{\prime}$ contains all points of $\mathcal{P}$.

Proposition 1.1 : ( Bumcrot, [4] ) Any finite linear space satisfying the following condition :

$$
\begin{array}{ll}
\text { I } & r_{m} \geq k_{M}+2 \\
\text { II } & k_{m}\left(k_{m}-1\right) \geq r_{M}
\end{array}
$$

is a hyperbolic plane in the sense of Graves [6].

A linear space $\mathcal{S}=(\mathcal{P}, \mathcal{L})$ is said to be embeddable in a linear space $\mathcal{S}^{\prime}=\left(\mathcal{P}^{\prime}, \mathcal{L}^{\prime}\right)$ if $\mathcal{S}^{\prime}$ can be obtained from $\mathcal{S}$ by addition of some points called as ideal points and some lines called as ideal lines. 


\section{Main Results}

In this section, we showed that a linear space, which is the complement of linear space whose points are not on a pentagon, hexagon or a heptagon in a projective subplane of order $m$, is embeddable in an unique way in a projective plane of order $n$. In addition, we determined that this linear space is the complement of certain regular hyperbolic plane in the sense of Graves [6] with respect to a finite projective plane.

Proposition 2.1 : Any $(m+1)$-regular linear space with line degree $m-2$, $m-3$ or $m-4, m \geq 7$, is a hyperbolic plane in the sense of Graves [6] .

Proof : Let $\mathcal{S}$ be an $(m+1)$-regular linear space with line degree $m-2, m-3$ or $m-4$. It is clear that $r_{m} \geq k_{M}+2$ and

$k_{m}\left(k_{m}-1\right)=(m-4)(m-5) \geq m+1$, since $k_{m}=m-4, k_{M}=m-2$ and $r_{m}=r_{M}=m+1, m \geq 7$. By the Proposition $1.1, \mathcal{S}$ is a hyperbolic plane and it is called a hyperbolic plane of $(5, m)$-type.

Proposition 2.2 : A real complement of linear space whose points are on a pentagon in a projective of order $m$ is a hyperbolic plane, $m \geq 7$.

Proof : Let $\pi$ be a projective plane of order $m$ and $\mathcal{S}$ be a real complement of a pentagon in a projective plane of order $m$. The total number of points on a pentagon is $5(m-1)$. Thus, $\mathcal{S}$ is an $(m+1)$-regular linear space with $m^{2}-4 m+6$ points, $m^{2}+m-4$ lines and it's line degree is $m-2, m-3$, or $m-4$. Also, $\mathcal{S}$ is a hyperbolic plane of $(5, m)$-type by the Proposition 2.1 .

Proposition 2.3 : Any $(m+1)$-regular linear space with line degree $m-2, m-$ $3, m-4$ or $m-5, m \geq 9$, is a hyperbolic plane in the sense of Graves [6].

Proof : Let $\mathcal{S}$ be an $(m+1)$-regular linear space with line degree $m-2, m-$ $3, m-4$ or $m-5$. It is clear that $r_{m} \geq k_{M}+2$ and

$k_{m}\left(k_{m}-1\right)=(m-5)(m-6) \geq m+1$, since $k_{m}=(m-5), k_{M}=(m-2)$ and $r_{m}=r_{M}=m+1, m \geq 9$. By the Proposition $1.1, \mathcal{S}$ is a hyperbolic plane and it is called a hyperbolic plane of $(6, m)$-type.

Proposition 2.4 : A real complement of a linear space whose points are on a hexagon in a projective plane of order $m$ is a hyperbolic plane, $m \geq 9$.

Proof : Let $\pi$ be a projective plane of order $m$ and $\mathcal{S}$ be a real complement of a hexagon in $\pi, m \geq 9$. The total number of points on a hexagon is $6 m-9$. Thus; $\mathcal{S}$ is an $(m+1)$-regular linear space with $m^{2}-5 m+10$ points, $m^{2}+m-5$ lines and it's line degree is $m-2, m-3, m-4$ or $m-5$. Also, $\mathcal{S}$ is a hyperbolic plane of $(6, m)$-type by the Proposition 2.3 .

Proposition 2.5 : Any $(m+1)$-regular linear space with line degree $m-3, m-$ $4, m-5$ or $m-6, m \geq 10$, is a hyperbolic plane in the sense of Graves [6].

Proof : Let $\mathcal{S}$ be an $(m+1)$-regular linear space with line degree $m-3, m-$ $4, m-5$ or $m-6$. It is clear that $r_{m} \geq k_{M}+2$ and

$k_{m}\left(k_{m}-1\right)=(m-6)(m-7) \geq m+1$, since $k_{m}=(m-6), k_{M}=m-3$ and $r_{m}=r_{M}=m+1, m \geq 10$. By the Proposition 1.1, $\mathcal{S}$ is a hyperbolic plane and it is called a hyperbolic plane of $(7, m)$-type. 
Proposition 2.6 : A real complement of a linear space whose points are on a heptagon in a projective plane of order $m$ is a hyperbolic plane, $m \geq 10$.

Proof : Let $\pi$ be a projective plane of order $m$ and $\mathcal{S}$ be a real complement of a heptagon in $\pi, m \geq 10$. The total number of points on a heptagon is $7 m-14$. Thus; $\mathcal{S}$ is an $(m+1)$-regular linear space with $m^{2}-6 m+15$ points, $m^{2}+m-6$ lines and it's line degree is $m-3, m-4, m-5$ or $m-6$. Also, $\mathcal{S}$ is a hyperbolic plane of $(7, m)$-type, by the Proposition 2.5 .

Theorem 2.1 : Let $\mathcal{S}=(\mathcal{P}, \mathcal{L})$ be an $(n+1)$-regular linear space such that:

(i) $v=n^{2}+n-\left(m^{2}-4 m+5\right), b=n^{2}+n+1, m \geq 7, n \geq m^{2}, n, m \in \mathbb{Z}$.

(ii) $b_{n+3-m}=15$ and $b_{n+4-m}=10(m-4)$

(iii) every line has $n+1, n, n+3-m, n+4-m, n+5-m$ points.

Then $\mathcal{S}$ is embeddable in a unique way in a projective plane of order $n$ and is complement of a hyperbolic plane $(5, m)$-type.

Proof : Let $\mathcal{P}_{i j k}$ be the set of points of $\mathcal{S}$ such that there are $i$ lines of degree $n+3-m, j$ lines of degree $n+4-m, k$ lines of degree $n+5-m, h$ lines of degree $n$ and $w$ lines of degree $n+1$ through every point $P$ of it. Then;

$$
\begin{gathered}
(n+2-m) i+(n+3-m) j+(n+4-m) k+(n-1) h+w n=v-1 \\
i+j+k+h+w=n+1
\end{gathered}
$$

From the above equalities, the following results are obtained.

$$
\begin{aligned}
h & =\left(m^{2}-4 m+6\right)-(m-2) i-(m-3) j-(m-4) k \\
w & =n+1-\left(m^{2}-4 m+6\right)+(m-3) i+(m-4) j+(m-5) k
\end{aligned}
$$

Also; by the simple counting methods,

$$
\begin{aligned}
\sum_{i}\left|\mathcal{P}_{i}\right|= & v, \quad \sum_{t} b_{t}=b, \quad t \in\{n+1, n, n+3-m, n+4-m, n+5-m\} \\
& \sum_{i, j, k}\left|\mathcal{P}_{i j k}\right| i=15(n+3-m) \\
& \sum_{i, j, k}\left|\mathcal{P}_{i j k}\right| j=10(m-4)(n+4-m) \\
& \sum_{i, j, k}\left|\mathcal{P}_{i j k}\right| k=b_{n+5-m}(n+4-m) \\
& \sum_{i, j, k}\left|\mathcal{P}_{i j k}\right| h=n b_{n} \text { and } \sum_{i, j, k}\left|\mathcal{P}_{i j k}\right| w=(n+1) b_{n+1} .
\end{aligned}
$$


Then; the following results are obtained.

$$
\begin{aligned}
b_{n} & =\left(m^{2}-4 m+6\right)(n-m) \\
b_{n+1} & =n^{2}-m^{2}(n+5-m)+m(4 n+5)-5(n+1) \\
b_{n+3-m} & =15 \\
b_{n+4-m} & =10(m-4) \\
b_{n+5-m} & =\left(m^{2}-9 m+21\right)
\end{aligned}
$$

Since $b_{n}=\left(m^{2}-4 m+6\right)(n-m), n \geq m^{2}$ and $m \geq 7$, there exists at least one $n$-line. For every $n$-line $l$, we define

$$
\Pi_{l}=\{l\} \cup\{x: x \text { is a line disjoint to } l\}
$$

Since each point has degree $n+1=v(l)+1$, each point outside $l$ lies on exactly one line which is parallel to $l$. This shows that $\Pi_{l}$ is a partition of the points of $\mathcal{S}$ into disjoint lines, and $\Pi_{l}$ induces an equivalence relation among the lines in $\mathcal{S}$ of size $n$. This equivalent relation on the lines of size $n$ is refered as parallelism. Since $l$ meets $n^{2}$ other lines, $\left|\Pi_{l}\right|=n+1$. Hence; each $n$-line induces a partition of the points into $n+1$ lines refered as the parallel class associated with that $n$-line.

Suppose that $l$ and $l^{\prime}$ are two different $n$-lines which meet. Then $l^{\prime}$ meets $n$ lines of $\Pi_{l}$, so $\left|\Pi_{l} \cap \Pi_{l^{\prime}}\right|=1$.

Let each such parallel class corresponds to a " new point ". Consider the structure $\mathcal{S}^{*}=\left(\mathcal{P}^{*}, \mathcal{L}^{*}\right)$, where $\mathcal{P}^{*}$ is $\mathcal{P}$ along with the new points, and $\mathcal{L}^{*}$ consists of the lines of $\mathcal{L}$ " extended " by those parallel classes to which they belong. We first of all prove that $\mathcal{S}^{*}$ is a linear space. It is clear that two old points ( points of $\mathcal{P}$ ) are on a unique line of $\mathcal{L}^{*}$. Let $X$ and $Y$ be distinct new points. We show that they determine a unique line of $\mathcal{L}^{*}$. Let $l_{X}$ and $l_{Y}$ be $n$-lines which determine the parallel classes corresponding to $X$ and $Y$. If $l_{X}$ and $l_{Y}$ do not meet, then $X=Y$ which is a contradiction. So $l_{X}$ and $l_{Y}$ meet. Since the point degree of $\mathcal{S}$ is $n+1$, each point of $l_{Y}$ is on a unique line of the parallel class determined by $l_{X}$. This leaves precisely one line of the parallel class parallel to both $l_{X}$ and $l_{Y}$. This is the required line. It follows from our method of construction that each point of $\mathcal{S}^{*}$ is on $n+1$ lines.

Finally, it is shown that any two lines of $\mathcal{S}^{*}$ always meet. Let $l$ and $l^{\prime}$ be lines of $\mathcal{S}^{*}$ which do not meet in $\mathcal{S}$. Then neither $l$ or $l^{\prime}$ are $(n+1)$-lines. To prove that they meet in $\mathcal{S}^{*}$, it suffices to find an $n$-line parallel to both.

Let $l$ and $l^{\prime}$ be two disjoint lines which have size less than $n$ in $\mathcal{S}$. It is clear that $d(l)=n+1-v(l) \geq m-4$ and $d\left(l^{\prime}\right)=n+1-v\left(l^{\prime}\right) \geq m-4$ since $v(l) \in$ $\{n+1, n, n+3-m, n+4-m, n+5-m\}$, for all $l \in \mathcal{L}$.

Let $x$ be the number of lines meeting $l$ (excluding $l$ itself); let $y$ be the number of lines meeting $l^{\prime}$ ( excluding $l^{\prime}$ itself ); and $z$ be the number of lines meeting both 
$l$ and $l^{\prime}$. The following three equations are obtained by a simple counting method:

$$
\begin{aligned}
& x=n(n+1-d(l)) \\
& y=n\left(n+1-d\left(l^{\prime}\right)\right)
\end{aligned}
$$

and

$$
z=(n+1-d(l))\left(n+1-d\left(l^{\prime}\right)\right) .
$$

Therefore; $x+y-z=n^{2}-(d(l)-1)\left(d\left(l^{\prime}\right)-1\right)$.

Let $m\left(l, l^{\prime}\right)$ and $m_{n}\left(l, l^{\prime}\right)$ be the total number of lines and $n$-lines, respectively, meeting $l$ or $l^{\prime}$ excluding $l$ and $l^{\prime}$ themselves. Since; $m\left(l, l^{\prime}\right)=x+y-z$, the following result is obtained.

$$
m\left(l, l^{\prime}\right)=n^{2}-(d(l)-1)\left(d\left(l^{\prime}\right)-1\right) \leq n^{2}-(m-5)^{2} .
$$

Since any line of size $n+1$ meets every other line, all the lines of size $n+1$ meet both $l$ and $l^{\prime}$. Therefore; since $b_{n+1} \geq 0, d(l) \geq m-4$ and $d\left(l^{\prime}\right) \geq m-4$, it is clear that $m_{n}\left(l, l^{\prime}\right) \leq n^{2}-(m-5)^{2}-b_{n+1} \leq b_{n}$. Thus; there is at least one $n$-line parallel to both. Consequently; $\mathcal{S}^{*}$ is a projective plane of order $n$.

Consider the complement of $\mathcal{S}$ in $\mathcal{S}^{*} . \mathcal{S}^{*} \backslash \mathcal{S}$ is an $(m+1)$-regular linear space whose lines are set of $\{m-2\},\{m-3\}$ or $\{m-4\}$ points, which are extensions of $(n+3-m)$-lines, $(n+4-m)$-lines and $(n+5-m)$-lines of $\mathcal{S}$, respectively. Therefore; $\mathcal{S}^{*} \backslash \mathcal{S}$ is a hyperbolic plane of $(5, m)$-type, by the Proposition 2.1.

Theorem 2.2 : Let $\mathcal{S}=(\mathcal{P}, \mathcal{L})$ be an $(n+1)$-regular linear space such that:

(i) $v=n^{2}+n-\left(m^{2}-5 m+9\right), b=n^{2}+n+1, m \geq 9, n \geq m^{2}, n, m \in \mathbb{Z}$.

(ii) $b_{n+4-m}=45-3 b_{n+3-m}$ and $b_{n+5-m}=15(m-7)+3 b_{n+3-m}$

(iii) every line has $n+1, n, n+3-m, n+4-m, n+5-m, n+6-m$ points.

Then $\mathcal{S}$ is embeddable in a unique way in a projective plane of order $n$ and is complement of a hyperbolic plane $(6, m)$-type.

Proof : Let $\mathcal{P}_{i j k t}$ be the set of points of $\mathcal{S}$ such that there are $i$ lines of degree $n+3-m, j$ lines of degree $n+4-m, k$ lines of degree $n+5-m, t$ lines of degree $n+6-m, h$ lines of degree $n$ and $w$ lines of degree $n+1$ through every point $P$ of it. Then;

$$
\begin{gathered}
(n+2-m) i+(n+3-m) j+(n+4-m) k+(n+5-m) t+(n-1) h+w n=v-1 \\
i+j+k+t+h+w=n+1
\end{gathered}
$$

From the above equalities, the following results are obtained.

$$
\begin{aligned}
h & =\left(m^{2}-5 m+10\right)-(m-2) i-(m-3) j-(m-4) k-(m-5) t \\
w & =n+1-\left(m^{2}-5 m+10\right)+(m-3) i+(m-4) j+(m-5) k+(m-6) t
\end{aligned}
$$

Also; by the simple counting methods,

$$
\sum_{i}\left|\mathcal{P}_{i}\right|=v, \quad \sum_{t} b_{t}=b, \quad t \in\{n+1, n, n+3-m, n+4-m, n+5-m, n+6-m\}
$$




$$
\begin{aligned}
\sum_{i, j, k}\left|\mathcal{P}_{i j k t}\right| i & =(n+3-m) b_{n+3-m} \\
\sum_{i, j, k}\left|\mathcal{P}_{i j k t}\right| j & =\left(45-3 b_{n+3-m}\right)(n+4-m) \\
\sum_{i, j, k}\left|\mathcal{P}_{i j k t}\right| k & =\left(15(m-7)+3 b_{n+3-m}\right)(n+5-m) \\
\sum_{i, j, k}\left|\mathcal{P}_{i j k t}\right| t & =(n+6-m) b_{n+6-m} \\
\sum_{i, j, k}\left|\mathcal{P}_{i j k}\right| h & =n b_{n} \text { and } \sum_{i, j, k}\left|\mathcal{P}_{i j k}\right| w=(n+1) b_{n+1} .
\end{aligned}
$$

Then; the following results are obtained.

$$
\begin{aligned}
b_{n} & =\left(m^{2}-5 m+10\right)(n-m) \\
b_{n+1} & =n^{2}-m^{2}(n+6-m)+m(5 n+9)-9 n+6 \\
b_{n+4-m} & =45-3 b_{n+3-m} \\
b_{n+5-m} & =15(m-7)+3 b_{n+3-m} \\
b_{n+6-m} & =\left(m^{2}-14 m+55\right)-b_{n+3-m}
\end{aligned}
$$

Since $b_{n}=\left(m^{2}-5 m+10\right)(n-m), n \geq m^{2}$ and $m \geq 9$, there exists at least one $n$-line. For every $n$-line $l$, it can be defined $\Pi_{l}$ parallel classes and constructed $\mathcal{S}^{*}=\left(\mathcal{P}^{*}, \mathcal{L}^{*}\right)$ as in the proof of Theorem 2.1. Using the technique in the Theorem 2.1 , it is easily shown any two points of $\mathcal{S}^{*}$ are on exactly one line.

Thus, we must show that any two lines of $\mathcal{S}^{*}$ always meet. Let $l$ and $l^{\prime}$ be lines of $\mathcal{S}^{*}$ which do not meet in $\mathcal{S}$. Then neither $l$ or $l^{\prime}$ are $(n+1)$-lines. To prove that they meet in $\mathcal{S}^{*}$, it suffices to find an $n$-line parallel to both.

Let $l$ and $l^{\prime}$ be lines of $\mathcal{S}$ such that $v(l)<n$ and $v\left(l^{\prime}\right)<n$. It is clear that $d(l)=n+1-v(l) \geq m-5$ and $d\left(l^{\prime}\right)=n+1-v\left(l^{\prime}\right) \geq m-5$ since $v(l) \in\{n+$ $1, n, n+3-m, n+4-m, n+5-m, n+6-m\}$, for all $l \in \mathcal{L}$. Again using the technique in the Theorem 1.2 , it is easily calculated that $m_{n}\left(l, l^{\prime}\right) \leq n^{2}-(m-6)^{2}-b_{n+1} \leq b_{n}$, since $\min _{l \in \mathcal{L}}(n+1-v(l))=m-5$ and $n \geq m^{2}$. There is at least one $n$-line parallel to both. Thus; $\mathcal{S}^{*}$ is a projective plane of order $n$.

Consider the complement of $\mathcal{S}$ in $\mathcal{S}^{*} . \mathcal{S}^{*} \backslash \mathcal{S}$ is an $(m+1)$-regular linear space whose lines are set of $\{m-2\},\{m-3\},\{m-4\}$ or $\{m-5\}$ points, which are extensions of $(n+3-m)$-lines, $(n+4-m)$-lines, $(n+5-m)-$ lines and $(n+6-$ $m)$-lines of $\mathcal{S}$, respectively. Therefore; $\mathcal{S}^{*} \backslash \mathcal{S}$ is a hyperbolic plane of $(6, m)$-type, by the Proposition 2.3.

Theorem 2.3 :Let $\mathcal{S}=(\mathcal{P}, \mathcal{L})$ be an $(n+1)$-regular linear space such that:

(i) $v=n^{2}+n-\left(m^{2}-6 m+14\right), b=n^{2}+n+1, m \geq 10, n \geq m^{2}, n, m \in \mathbb{Z}$.

(ii) $b_{n+5-m}=105-3 b_{n+4-m}$ and $b_{n+6-m}=21(m-11)+3 b_{n+4-m}$ 
(iii) every line has $n+1, n, n+4-m, n+5-m, n+6-m, n+7-m$ points.

Then $\mathcal{S}$ is embeddable in a unique way in a projective plane of order $n$ and is complement of a hyperbolic plane $(7, m)$-type.

Proof : Let $\mathcal{P}_{i j k t}$ be the set of points of $\mathcal{S}$ such that there are $i$ lines of degree $n+4-m, j$ lines of degree $n+5-m, k$ lines of degree $n+6-m, t$ lines of degree $n+7-m, h$ lines of degree $n$ and $w$ lines of degree $n+1$ through every point $P$ of it. Then;

$$
\begin{gathered}
(n+3-m) i+(n+4-m) j+(n+5-m) k+(n+6-m) t+(n-1) h+w n=v-1 \\
i+j+k+t+h+w=n+1
\end{gathered}
$$

From the above equalities, the following results are obtained.

$$
\begin{aligned}
h & =\left(m^{2}-6 m+15\right)-(m-3) i-(m-4) j-(m-5) k-(m-6) t \\
w & =n+1-\left(m^{2}-6 m+15\right)+(m-4) i+(m-5) j+(m-6) k+(m-7) t
\end{aligned}
$$

Also; by the simple counting methods,

$$
\begin{aligned}
\sum_{i}\left|\mathcal{P}_{i}\right|=v, \quad \sum_{t} b_{t}=b, \quad t & \in\{n+1, n, n+4-m, n+5-m, n+6-m, n+7-m\} \\
\sum_{i, j, k, t}\left|\mathcal{P}_{i j k t}\right| i & =(n+4-m) b_{n+4-m} \\
\sum_{i, j, k, t}\left|\mathcal{P}_{i j k t}\right| j & =\left(105-3 b_{n+4-m}\right)(n+5-m) \\
\sum_{i, j, k, t}\left|\mathcal{P}_{i j k t}\right| k & =\left(21(m-11)+3 b_{n+4-m}\right)(n+6-m) \\
\sum_{i, j, k, t}\left|\mathcal{P}_{i j k t}\right| t & =(n+7-m) b_{n+7-m} \\
\sum_{i, j, k}\left|\mathcal{P}_{i j k}\right| h & =n b_{n} \text { and } \sum_{i, j, k}\left|\mathcal{P}_{i j k}\right| w=(n+1) b_{n+1} .
\end{aligned}
$$

Then; the following results are obtained.

$$
\begin{aligned}
b_{n} & =\left(m^{2}-6 m+15\right)(n-m) \\
b_{n+1} & =n^{2}-m^{2}(n+7-m)+m(6 n+14)-(14 n-7) \\
b_{n+5-m} & =105-3 b_{n+4-m} \\
b_{n+6-m} & =21(m-11)+3 b_{n+4-m} \\
b_{n+7-m} & =\left(m^{2}-20 m+120-3 b_{n+4-m}\right)
\end{aligned}
$$

Since $b_{n}=\left(m^{2}-6 m+15\right)(n-m), n \geq m^{2}$ and $m \geq 10$ there exists at least one $n$-line. For every $n$-line $l$, it can be defined $\Pi_{l}$ parallel classes and constructed $\mathcal{S}^{*}=\left(\mathcal{P}^{*}, \mathcal{L}^{*}\right)$ as in the proof of Theorem 2.1. Using the technique in the Theorem 2.1 , it is easily shown any two points of $\mathcal{S}^{*}$ are on exactly one line. 
Thus, we must show that any two lines of $\mathcal{S}^{*}$ always meet. Let $l$ and $l^{\prime}$ be lines of $\mathcal{S}^{*}$ which do not meet in $\mathcal{S}$. Then neither $l$ or $l^{\prime}$ are $(n+1)$-lines. To prove that they meet in $\mathcal{S}^{*}$, it suffices to find an $n$-line parallel to both.

Let $l$ and $l^{\prime}$ be lines of $\mathcal{S}$ such that $v(l)<n$ and $v\left(l^{\prime}\right)<n$. It is clear that $d(l)=n+1-v(l) \geq m-6$ and $d\left(l^{\prime}\right)=n+1-v\left(l^{\prime}\right) \geq m-6$ since $v(l) \in\{n+$ $1, n, n+4-m, n+5-m, n+6-m, n+7-m\}$, for all $l \in \mathcal{L}$. Again using the technique in the Theorem 1.2, it is easily calculated that $m_{n}\left(l, l^{\prime}\right) \leq n^{2}-(m-7)^{2}-b_{n+1} \leq b_{n}$, since $\min _{l \in \mathcal{L}}(n+1-v(l))=m-6$ and $n \geq m^{2}$. There is at least one $n$-line parallel to both. Thus; $\mathcal{S}^{*}$ is a projective plane of order $n$.

Consider the complement of $\mathcal{S}$ in $\mathcal{S}^{*} . \mathcal{S}^{*} \backslash \mathcal{S}$ is an $(m+1)$-regular linear space whose lines are set of $\{m-3\},\{m-4\},\{m-5\}$ or $\{m-6\}$ points, which are extensions of $(n+4-m)$-lines, $(n+5-m)$-lines, $(n+6-m)$-lines and $(n+7-$ $m)$-lines of $\mathcal{S}$, respectively. Therefore; $\mathcal{S}^{*} \backslash \mathcal{S}$ is a hyperbolic plane of $(7, m)$-type, by the Proposition 2.5.

ÖZET:Bu çalışmada, $m$. mertebeden projektif altdüzlemdeki beşgen, altıgen ve yedigene ait olmayan noktaları noktalar kümesi olarak kabul eden bir lineer uzayın tümleyeni olan ve $n$ mertebeden projektif düzleme tek olarak gömülebilen lineer uzaylar üzerinde çalışılmıştır. Ayrıca, bu lineer uzayın projektif düzlemlere göre Graves anlaminda regular hiperbolik düzlemin tümleyeni olduğu gösterilmiştir.

\section{REFERENCES}

[1] Anapa, P and Günaltılı, I .: On the embedding of complements of some hyperbolic planes III, Ars Combinatoria 86 ( 2008)

[2] Batten, L.M .; Embedding pseudo-complements in finite projective planes, Ars Combinatoria 24 (1987), 129-132.

[3] Batten, L.M and Beutelspacher, A.; The theory of finite linear space, Cambridge University Press, New-York-Melbourne, ( 1993)

[4] Bose, R.C and Shrikhande, S.S .; Embedding the complement of an oval in a projective plane of even order, Discrete Math. 6. (1973), 305-312.

[5] Bumcrot, R.J .; Finite hyperbolic spaces, Atti Convegno Geom. comb e sue Appl. Perugia (1971), 113-130.

[6] Dickey, L. J .; Embedding the complement of a unital in a projective plane, Atti del convegno di Geometria combinatoria e sue Applicazioni, Perugia, 1971, 199-203.

[7] Graves, L. M .; A finite Bolyai-Lobachevsky plane, Amer. Math. Monthly 69 (1962), 130-132

[8] Günaltılı, İ. and Olgun, S .; On the embedding of some linear spaces in finite projective planes, J. Geom. 68 (2000) 96-99.

[9] Günaltılı, İ., Anapa, P. and Olgun, S .; On the embedding of complements of some hyperbolic planes, Ars Combinatoria 80 (2006 ), 205-214.

[10] Günaltılı, İ., Anapa, P. and Olgun, S .; On the embedding of complements of some hyperbolic planes II,( under riview in Discrete Mathematics ).

[11] Günaltılı, İ .; Pseudo-semicomplements in finite projective planes, ( accepted in Ars Combinatoria ). 
[12] Kaya, R. and Olgun, S .; Construction of some hyperbolic planes using Baer subplanes, Combinatoric's 88. Verlang : Mediterranean Press, 105-112.

[13] Kaya, R. and Ozcan, E .; On the construction of Bolyai-Lobachevsky planes from projective planes, Rendiconti del Seminario Mathematico di Brescia 7 (1984), 427-434.

[14] Ralston, T .; On the embeddability of the complement of a complete triangle in a finite projective plane. Ars Combinatoria 11 (1981 ) 271-274.

[15] Ostrom, T.G .; Ovals and finite Bolyai-Lobachevsky Planes, Amer. Math. Monthly, 69 (1962), 899-901.

[16] Özcan, E., Olgun, S. and Kaya, R .; On the line classes in some finite hyperbolic planes, Commun. Fac.Sci.Univ.Ank.Series A, V38, (1989), 7-13.

[17] Sandler, R .; Finite homogeneous Bolyai-Lobachevsky planes, Amer. Monthly 70 (1963), 853-854.

[18] Segre, B .; Ovals in a finite projective plane, Canada J. Math 7 (1955), 414-416.

Current address: (Eskişehir Osmangazi University, Department of Mathematics, EskişehirTURKEY

E-mail address: panapa@ogu.edu.tr, igunalti@ogu.edu.tr, solgun@ogu.edu.tr $U R L:$ http://math.science.ankara.edu.tr 


\section{INSTRUCTIONS TO CONTRIBUTORS}

Mathematics and Statistics of COMMUNICATIONS accepts original research articles written in English in the fields of Mathematics and Statistics. Review articles written by eminent scientists can also be invited by the Editor.

Three copies of the manuscripts must be submitted in AMS Article Tex format.

Formulas should be numbered consecutively in parentheses ( ) and each rnanuscript should be accompanied by classification numbers from the American Mathematical Society's classification scheme.

It is a fundamental condition that articles submitted to Communications have not been previously published and will not be simultaneously submitted or published elsewhere. After the manuscript has been accepted for publication, i.e., after referree-recommended revisions are complete, the author will not be permitted to rnake any new additions to the manuscript.

Before publication the galley proof is always sent to the author for correction. Thus it is solely the author's responsibility for any typographical mistakes which occur in their article as it appears in the Journal.

\section{Title Page:}

The title should not be long but should be informative. Each title page must contain (i) The title of the paper in English, (ii) The complete name(s) of the author(s), (iii) The name and the address of the University.

2. Abstract :

The abstract should not exceed 200 words and it should condense the essential features of the articles with the focus on the major advances in the field.

\section{References :}

References must be listed in alphabetical order at the end of the article and then numbered in brackets [1]. Within the manuscript, refer to the references by their given number, again in brackets [1]. They should be styled and punctuated according to the following examples:

[1] Kelley, J.L., General Topology, 1970, New York: Van Nostrand.

[2] Maddox,1.J., Some incusion theorems, Proc. Glasgow Math. Assn. 6 (1964),

161-168.

Abstracts, unpublished data and personal communications should not be given in the references but they may be mentioned in the text.

\section{Footnotes:}

Footnotes, except a possible acknowledgement of assistance or financial support on the first page, should be avoided by being incorparated into the text. 


\section{Tables and Figures:}

All tables and figures must be numbered consecutively throughout the paper ( Table 1, Figure 2) and also have a caption or legend.

6. Acknowledgements:

Acknowledgements should be given as short as possible at the end of the text.

7. Reprints:

25 free reprints will be provided for each paper. Irrespective of their acceptance, manuscripts will not be returned to the authors.

\section{Computer Disk:}

After the acceptance of manuscripts for publication, we will ask you to submit a revised electronic copy of the manuscript which is written in AMS tex format. For electronic submission please visit our WEB page at http://math.science.ankara.edu.tr/dergi/dergi.htm

\section{Charges:}

Each paper is due to be charged for the amount of which is determined by the administiration each year.

\section{Address:}

Text should be sent to the following address:

Prof.Dr. Öner ÇAKAR- Editor-in-Chief, Communications

Ankara University, Faculty of Sciences

06100, Tandoğan, ANKARA - TURKEY 